\title{
Imagen social de las bebidas alcohólicas
}

\author{
Pascual Pastor, f.
}

Unidad de Alcohología. Alcoi. Generalitat Valenciana.

Enviar correspondencia: Francisco Pascual Pastor. Unidad de Alcohologia. C/ El Camí, 40. 03802 Alcoi.

Tfn. 965543047. E-mail: fr.pascualp@coma.es

\section{RESUMEN}

Se estudia la percepción que tiene la sociedad de las bebidas alcohólicas, mediante un análisis de encuestas relacionadas con este aspecto y de una búsqueda en hemeroteca de los diarios de más tirada nacional, así como de otras fuentes fidedignas.

El alcohol forma parte de nuestra vida social y cultural y en general se observa una excesiva permisividad en el consumo incluso abusivo de bebidas alcohólicas. Pero al mismo tiempo se rechaza todo lo que suena a alcoholismo. Por un lado se intenta otorgar a las bebidas alcohólicas, en concreto a la cerveza y al vino, un valor nutritivo e incluso protector de la salud, y por otro se observa un rechazo social al abuso, aunque existen dificultades para concretar lo que significa abuso.

Todavía persisten las falsas creencias populares que otorgan al alcohol una función estimulante, calórica, nutritiva y con capacidad para dar fuerzas y aumentar el apetito sexual. Existen importantes campañas publicitarias con intereses económicos más o menos encubiertos que ayudan a mantener estas ideas.

Las bebidas alcohólicas siguen preocupando más a los políticos y a los profesionales que a la población en general, que lo sienten más como un instrumento más en nuestras vidas que va ligado sobre todo a momentos de relajación, diversión, compañía o soledad.

Palabras clave: Bebidas alcohólicas, percepción social, alcohol, alcoholismo, sociedad.

\begin{abstract}
The perception that society has of alcoholic drinks is studied through an analysis of surveys on this subject and a search of the libraries of the biggest circulation national newspapers in addition to other reliable sources.

Alcohol forms part of our cultural culture and, in general, an excessive permissiveness to the consumption -even an abusive one- of alcoholic drinks is to be observed. But, at the same time, everything redolent of alcoholism is rejected. On the one hand, there is an endeavour to bestow a nutritive value, even to protecting one's health, on beer and wine in particular, and on the other, a significant social rejection of abuse is to be seen although there are difficulties in defining what abuse signifies.

False popular beliefs that give alcohol a stimulant, calorific and nutritive function and with the ability to provide strength and to increase sexual appetite still persist. Important publicity campaigns with more or less concealed economic interests assist in maintaining these ideas.

Alcoholic beverages continue to preoccupy politicians and professionals more that the general public who feel it to be one instrument more in their lives, associated most of all with times of relaxation, fun, company or solitude.
\end{abstract}

Key words: Alcoholic drinks, social perception, alcohol, alcoholism, society.

tural y gastronómico consolidado dentro de las costumbres sociales. Últimamente se observa además una excesiva tolerancia también para los consumos de abuso, sobre todo cuando el abuso está ligado a situaciones de fiesta o diversión. 
Esta permisividad, no obstante, no es ajena a los graves problemas que pueden desencadenar ciertos consumos abusivos: accidentes de tráfico, reyertas, problemas familiares, intoxicaciones etílicas... Este hecho hace más difícil si cabe la ya de por sí ardua tarea de aproximación y diagnóstico de cualquier individuo que presente una dependencia alcohólica, ya que es complicado para cualquier personas admitir que ha sobrepasado los límites de la "normalidad" por lo que creará en él férreos mecanismos de resistencia.

En general se es consciente de los graves problemas que conlleva el alcoholismo pero no de los riesgos de las situaciones de abuso.

Se han desarrollado para ello distintas campañas preventivas, que en la mayoría de los casos no han obtenido el resultado esperado y es que existe por otro lado una serie de intereses comerciales que además repercuten directamente sobre las arcas del estado, muy difíciles de contrarrestar. De hecho una de las muestras patentes es la excesiva permisividad y la agresividad de la publicidad de las bebidas alcohólicas.

Incluso a nivel formativo, entre los profesionales sanitarios se observa una clara carencia curricular sobre este tema, lo que hará que el abordaje de los problemas derivados del consumo de bebidas alcohólicas o bien no se haga $\mathrm{o}$, si se hace, el profesional adolezca de muchas limitaciones.

Un estudio realizado en Murcia (Rodríguez Fernández et al.) (1) entre médicos de atención primaria y con un total de 227 encuestas válidas, concluyó que un 66'5\% poseían unos conocimientos apropiados sobre el consumo excesivo de alcohol y problemas derivados. Por áreas temáticas, es insuficiente el discernimiento de los conceptos de bebedor excesivo y consumidor de riesgo.

El médico tiende a fijarse en las secuelas físicas del consumo de alcohol, en detrimento de cumplimentar una historia clínica que contemple los consumos y de recabar información sobre los factores psicosociales que puedan estar implicados. Concluyendo que a pesar de tener bastante información, esta es inadecuada.

En la conferencia ministerial de Estocolmo, se dijo que en el mundo existen 140 millones de personas con dependencia al alcohol, sólo en EE.UU. el costo económico del consumo excesivo de bebidas alcohólicas se elevó a 53 mil millones de dólares en 1996 (según el Departamento americano de Justicia) (2).

Estos datos deben ser contrarrestados con políticas de salud, pero cualquier medida que se tome debe "contar con el apoyo popular basado en la comprensión sobre su importancia". "El alcohol está tan profundamente incluido en nuestra cultura y actividades sociales, que en ocasiones las políticas pro salud encuentran fuertes oposiciones" (2).

\section{LA SITUACIÓN EN ESPAÑA}

España era el $5^{\circ}$ consumidor de bebidas alcohólicas del mundo por detrás de Luxemburgo, Francia, Portugal y Hungría según los datos del World Drinks Trends del año 1996 (3), en estos momentos ocupamos el $7^{\circ}$ puesto con un consumo medio anual per capita de 10'1 litros de alcohol.

Según el Plan Nacional sobre Drogas el alcohol es la sustancia tóxica más consumida por los españoles, aunque según los últimos datos su consumo no parece estar aumentando (4). No obstante en España durante 1998 se llegaron a vender, 1.414'74 millones de litros de vino, 2.153'59 millones de litros de cerveza, 71'25 millones de litros de sidra y 173'92 millones de litros de licores.

La bebida más consumida en nuestro país será según los datos anteriores, la cerveza. Ocupamos el nada despreciable octavo lugar en el consumo de cerveza por habitante y año con una cantidad de 77'1 litros.

Últimamente en los suplementos dominicales de algunos periódicos de tirada nacional aparecen publirreportajes ensalzando el valor nutritivo de la cerveza, avalado por estudios 
de distintas Universidades, incidiendo en el bajo contenido alcohólico.

La cerveza se ha intentado vender como un producto de alto nivel nutritivo, aportado por la cebada y la levadura, pero no es así. Un vaso de cerveza proporciona cantidades de proteínas, hierro y vitaminas $B_{1}$ y $B_{2}$. En cambio nos da una excesiva cantidad de calorías (5).

Tradicionalmente se ha relacionado el consumo de cerveza con efectos beneficiosos durante el embarazo y en el período de lactancia. Según diversos estudios (6-7) aproximadamente el $24 \%$ de las embarazadas son consumidoras de riesgo. El alcohol pasa la barrera placentaria, provoca el síndrome alcohólico fetal, que en estos momentos se ha situado en una incidencia de 2 para cada 1000 recién nacidos vivos, la misma que el síndrome de Down. En ningún artículo de los antes mencionados se advierte del riesgo del consumo de bebidas alcohólicas.

La publicidad de bebidas alcohólicas no sólo se da en la prensa escrita. Las televisiones tanto públicas como privadas ofrecen publicidad indirecta de bebidas alcohólicas con más de 20 grados, algo que está prohibido por la legislación vigente. La publicidad del alcohol aparecía en las series españolas en 1999 hasta en un $87 \%$ de los casos. La publicidad está presente en acontecimientos deportivos, programas musicales e incluso en los cómics.

Para el Ministerio de Agricultura, Pesca y Alimentación el gasto por hogar en bebidas alcohólicas en el año 1999 representa un 4\% del total asignado a la alimentación, esta cifra aumenta hasta un $32 \%$ en hostelería y restauración (8). El consumo diario y por lo tanto más problemático se centra sobre todo en los mayores de 49 años (4).

Hay otros datos significativos a la hora de valorar el problema y estos son los relacionados con los accidentes de tráfico. En 1998 se analizaron un total de 1.245 fallecidos en accidentes de tráfico, estableciéndose una importante relación con el consumo de alcohol. En un $51 \%$ se corroboró un consumo de alcohol. El 38\% sólo habían consumido alco- hol. El $43 \%$ de los fallecidos presentaban una tasa de alcoholemia positiva, superior a 0'8 en aquel momento (9). En 1999 fallecieron 2.500 jóvenes en accidente de tráfico por consumir drogas y alcohol. El $38 \%$ de los bebedores han sufrido algún accidente de tráfico y casi la mitad de los fallecidos en accidente de tráfico dan una alcoholemia positiva, superior a 0'5 en la actualidad (9).

A pesar de los datos ofrecidos, el consumo de alcohol en España está muy arraigado y debe ser considerado como parte de la vida cultural y social integrada por un conjunto de factores interactivos. Es un hábito que forma parte de los estilos de vida de los países occidentales. En una encuesta realizada por EDIS (10) sólo 1 de cada 4 ciudadanos lo mencionaban espontáneamente como una droga, y es que el concepto general que se tiene es el de una "sustancia que se puede controlar".

En 1995, en Europa su producción representó un $2 \%$ del PNB y los costes económicos que ocasionaron las consecuencias de este consumo alcanzó el $5-6 \%$ del PNB según EUROCARE (2).

Según la Federación de Alcohólicos Rehabilitados de España (F.A.R.E.) (11) el elevado consumo está determinado por:

-El alto prestigio del alcohol.

-La gran disponibilidad de las bebidas alcohólicas.

- La Carencia de restricciones legislativas.

“El factor decisivo es la aparición de nuevos estilos de vida, basados en la hegemonía de los ideales hedonistas y de las ambiciones económicas. Nuestro comportamiento social es competitivo y agresivo, y el consumo se ha convertido en el más alto valor" (11).

El 53\% de los españoles consideran "normal" beber hasta seis copas en una salida de fin de semana. Es ya toda una tradición. El $56 \%$ creen que seis copas al día no dañan la salud (12). El consumo de alcohol está sobrevalorado, como si de una proeza se tratase el hecho de aguantar grandes cantidades en la ingesta de bebidas alcohólicas, aunque se menosprecia la figura del mal entendido "borracho". 
Esta sobrevaloración nos lleva a situaciones en las que casi un $5 \%$ de los españoles tienen un consumo "problemático" de alcohol, más de la mitad lo beben, en torno al $15 \%$ reconocen consumirlo a diario y un $7^{\prime} 7 \%$ se excede los fines de semana. La consecuencia más inmediata son los más de 165.511(13) ingresos hospitalarios anuales debidos al alcohol.

El alcohol mata entre 20.000 (13) a 40.000 (14) españoles cada año, lo que representa de un $6 \%$ a un $12 \%$ de la mortalidad total. De ellos, en 10.000 casos el alcohol es la causa directa de muerte. El tema del alcohol es tan importante y a la vez tan controvertido que podemos encontrar cifras epidemiológicas tan dispares como las aquí presentadas teniendo en cuenta no solo los investigadores, sino la fuente de los datos que pueden ofrecer sesgos importantes según sus propios intereses. En Estados Unidos el número de muertes se eleva a 100.000. En 1999 incluso se llegó a proponer la expropiación del coche a los conductores borrachos (15).

El alcohol está relacionado con el $46 \%$ de los homicidios, el $25 \%$ de los suicidios y el $40 \%$ de los accidentes de tráfico (16). En España todo ello representa 224.000 años potenciales de vida perdidos, y un impacto económico de 637.717 millones por año o lo que es lo mismo el $16 \%$ del presupuesto de la sanidad pública (13).

Las complicaciones se extienden al ámbito familiar y existe una relación directa con los malos tratos, tanto es así que durante el año 2000 la Ministra de Sanidad española ha solicitado que el alcoholismo sea un agravante en los casos de malos tratos. Pidió a la sociedad que no vea esta adicción como una excusa para el mal trato femenino y que se haga todo lo posible para rehabilitar al enfermo (17).

De todas formas todavía existe un sector importante de la población que sigue creyendo en los falsos mitos del alcohol como una sustancia estimulante, que es buena para el corazón, que nos da fuerzas, que aumenta el deseo sexual, abre el apetito y proporciona alimento.
En una encuesta realizada por EDIS (10) en 1998 a padres con hijos de entre 12 a 14 años, dio como resultado que el 63 ' $8 \%$ de los entrevistados creían que el consumo de bebidas alcohólicas aportaba una serie de beneficios tanto físicos, como psicológicos y sociales. Entre los beneficios sobre el organismo destacaban: ayuda a la digestión, combate el frío, cura los catarros, favorece el crecimiento y tiene efectos estimulantes sobre la circulación sanguínea.

En este sentido hay que contrarrestar estas falsas informaciones con estudios que puedan desmentirlas, por ejemplo, para desmitificar el efecto cardioprotector del vino un estudio realizado por la American Heart Association reveló que el zumo de uva es tan sano como el vino tinto (15).

A nivel psicológico la percepción social indica que el alcohol da euforia, infunde valor, aumenta el tono vital y ayuda a tomar decisiones, no obstante se ha constatado que más de la mitad de los pacientes alcohólicos presentan síntomas depresivos asociados a su alcoholismo. El perfil de estos enfermos es el de un hombre de cuarenta años, casado y con trabajo, socialmente integrado y clínicamente recuperable.

En cuanto al área social se cree que el alcohol facilita las relaciones sociales, facilita el inicio de las relaciones sexuales, ayuda a superar las dificultades sociales y profesionales y estimula la creatividad intelectual.

En el mismo estudio se valoran las bebidas alcohólicas como importantes en la alimentación $(54 \%)$, en las costumbres sociales $\left(70{ }^{\prime} 6 \%\right)$ y en la economía para el país $\left(71^{\prime} 1 \%\right)$ y es que la omnipresencia de las bebidas alcohólicas en cualquier acto social lo hace poco menos que imprescindible.

Según UGT algunos de estos motivos justifican el gran porcentaje de consumidores en el ámbito laboral. Eso unido a jornadas laborales excesivamente largas, la exposición a temperaturas extremas, los turnos rotativos, el estrés o las tareas repetitivas conducen en ocasiones al consumo de alcohol y favorecen su abuso. 
Se calcula que dos millones de trabajadores beben en exceso y el $41^{\prime} 6 \%$ de los españoles con empleo conocen a algún compañero que consume alguna sustancia tóxica o bebe demasiado (15).

No existe ninguna profesión que esté exenta de estos riesgos, en el año 2000 las líneas aéreas holandesas impidieron volar a un piloto ebrio con una tasa de 2'2 gramos de alcohol en sangre además de ponerle una multa de 1.140 euros (unas 200.000 pesetas). $Y$ en el sentido contrario en el año 1999 IBERIA declaró "persona non grata" a cinco pasajeros que volaron borrachos, insultaron a los miembros de la tripulación y los agredieron físicamente.

El consumo de alcohol se asocia con cualquier actividad recreativa, los ecologistas pidieron en el año 2000 que se realizase el test de alcohol a los cazadores: "es habitual que antes de una jornada cinegética, el grupo se reúna a desayunar y se tome algunas copitas para entrar en calor. Son momentos que rozan la madrugada y cuando el frío aprieta los cazadores no tienen reparos en cargarse de alcohol". "En las monterías algunos cazadores llevan una petaca en la canana" (15). La Federación Madrileña de caza quiso desmentir tales acusaciones.

A partir de diversos estudios podemos concluir que los principales motivos aducidos por los españoles para justificar el consumo de bebidas alcohólicas son por orden de mayor a menor importancia: por gusto, diversión, influencia social, problemas de relación, aburrimiento por las condiciones de trabajo (10, 18-22).

\section{LA SITUACIÓN EN EUROPA}

Hay una preocupación creciente por parte de los adultos por el consumo de alcohol entre los jóvenes, que sufrió un importante incremento durante los años 80. Según Craplet (23) los adultos muestran un alto grado de hipocresía: mientras desaprueban el consumo de alcohol entre los jóvenes, ellos tie- nen una actitud muy indulgente hacia su propio consumo, desplegando argumentos de salud para su justificación.

Como parte del Plan de Acción Europeo sobre el Alcohol, la Conferencia de París (12 -14 Diciembre 1995) instó a todos los Estados miembros a elaborar políticas globales sobre el alcohol y a aplicar programas dependiendo de los diferentes entornos, culturales, sociales y económicos, se elaboró una carta de principios éticos y se consensuaron diez estrategias para la "Acción sobre el Alcohol".

La Carta Europea define básicamente el derecho a la educación, derecho al tratamiento y en general el derecho a poder vivir en un entorno libre de bebidas alcohólicas. Varios años después la región europea sigue teniendo las tasas mas altas de consumo de alcohol del mundo con una media de 7'3 litros por persona y año (1998) con grandes diferencias entre los distintos países que van de 13 '3 litros hasta 0'9 litros. Sobre todo se ha observado un incremento importante en los países del Este, donde existen 17 países con altos niveles de consumos (más de 10 litros por habitante y año) (2). Durante el decenio 1988-1998, once países han mantenido el consumo y trece incluso han logrado disminuirlo, otros 15 países lo han incrementado.

Cada bebida tiene su patrón de consumo y su zona de influencia. En Europa, el vino es la bebida alcohólica preferida en Francia, Portugal, España, Italia, Hungría Suiza y Rumania, Se consume en las comidas, se utiliza como un nutriente barato, el número de abstemios en estos países es bajo y un consumo moderado no está mal considerado. Los mitos se reproducen fidedignamente de unos países a otros otorgándole a las bebidas alcohólicas la misma suerte de virtudes.

Frente a este escenario, la cerveza es la favorita en Gran Bretaña, Irlanda, Luxemburgo, Bélgica, Dinamarca, Alemania, Austria, Chequia y Eslovaquia. Frente al vino esta bebida se consume entre las comidas, después del trabajo, en los actos sociales $y$, sobre todo, los fines de semana. 
Cuestión distinta son las bebidas destiladas. En los países consumidores de licores, Noruega, Finlandia, Islandia, Países Bajos, Polonia y la antigua Europa Oriental, el alcohol se usa como intoxicante y lo consume una escasa proporción de individuos, pero en cantidades muy elevadas. En estos países existe una actitud contraria y de rechazo al alcohol.

El 7\% de los adultos de Europa padecen alcoholismo (25). En Francia se calcula que 5 millones de franceses tienen problemas con el alcohol, de los cuales 2 millones son alcoholdependientes. (14) El coste derivado de las patologías ocasionadas por el alcohol se eleva a 14.000 - 17.000 millones de francos y la suma global de gastos derivados del alcohol llega a 80.000 millones de francos.

El $25 \%$ de las hospitalizaciones tienen por causa el alcohol, el alcohol provoca 40.000 muertos al año, un tercio de los accidentes de tráfico, el $20 \%$ de los accidentes domésticos, el $15 \%$ de los accidentes laborales y el $80 \%$ de las discusiones y violencia familiar. (14)

Como podemos observar los datos no difieren excesivamente de los españoles, así como tampoco difieren las motivaciones para beber: tentativa de escapar a la cotidianeidad considerada insoportable, tentativa de superar una inadaptación psicológica a la comunicación o de calmar cualquier sufrimiento psíquico, voluntad de transgredir la norma por revuelta o amor al riesgo y la presión del grupo de iguales.

Tampoco difiere la política de consumo y las estrategias publicitarias a través de los medios de comunicación o con el patrocinio de distintos eventos, culturales, sociales o deportivos. El papel de los medios de comunicación podría usarse en el sentido contrario, promoviendo hábitos saludables.

Rusia tiene 706 destilerías de Vodka, las estimaciones de consumo son de entre 10 a 18 litros de alcohol puro por persona y año (1999) El alcohol es la principal causa de muerte en Rusia, 2 de cada 3 mueren en estado de intoxicación etílica (26).
A nivel Europeo se debate también el riesgo - beneficio del consumo del alcohol en relación con la protección de las enfermedades cardiovasculares, llegando a la conclusión de que no existe ninguna justificación para dar un mensaje al público en general a favor del consumo moderado de alcohol. Esto haría un flaco favor a las políticas dirigidas hacia la restricción en el consumo de bebidas alcohólicas.

La intervención breve se ha mostrado muy efectiva para reducir el consumo entre los bebedores excesivos (27). Los bebedores problema también pueden beneficiarse de una intervención, aunque no presenten dependencia al alcohol, sobre todo trabajando con la motivación del paciente a partir del modelo desarrollado por Prochaska y Diclemente (28).

Otra reflexión a considerar es que también en Europa, los miembros de la familia son las víctimas olvidadas de los problemas del alcohol. Según la O.M.S. en Europa hay 10 millones de adultos y niños que sufren los efectos adversos del alcohol por tener algún bebedor en su familia (25). Sin embargo existen pocos programas bien definidos para atender a estas personas.

Se estima que al menos 4'5 millones de niños está creciendo en familias dañadas por el alcohol, las cifras reales pueden llegar a 7'7 millones (25). Se evidencia en estos niños un grave aislamiento social, viéndose obligados a asumir roles y responsabilidades de los adultos.

\section{DISCUSIÓN}

También en la conferencia ministerial de Estocolmo se debatió sobre las consecuencias del consumo de bebidas alcohólicas, señalando entre otras, las nefastas consecuencias personales, sociales, las lesiones de tráfico, los problemas en el hogar, los ahogamientos, los suicidios y los crímenes violentos además de las separaciones matrimoniales y de las repercusiones que puede causar 
en los hijos, ocasionando daños emocionales irreparables.

Se señaló la importancia que tiene el "counselling" dirigido a personas con consumos elevados de alcohol, obteniendo buenos resultados tanto sobre la forma como en la intensidad del consumo, produciendo una reducción del mismo.

Pero para poder realizar una política de salud correcta las personas necesitan entender que el consumo de alcohol no solo afecta a su propia salud y felicidad, sino que estas consecuencias se extienden entre las personas que se relacionan con ellas.

Ello lleva a considerar que se deben promover esfuerzos institucionales para sensibilizar a la sociedad de los riesgos reales que comporta el alcohol, de modo que la población en general pueda cambiar esa imagen tan "relajada" que tiene de las bebidas alcohólicas.

Según Comas (29) la declaración de la Conferencia sobre atención primaria de Alma Ata en 1978, así como las previsiones de la estrategia Mundial "Salud para todos en el año 2000" se saldaron con un sonoro fracaso, en gran medida como consecuencia de la presión de los agentes económicos vinculados al mercado del alcohol. La reducción del consumo de alcohol es un objetivo prioritario y pendiente no solo de la O.M.S., sino de todos los gobiernos europeos.

Para favorecer esta reducción habría que tener en cuenta los factores de riesgo para el consumo e incidir sobre ellos y no solo trabajar desde una perspectiva estrictamente sanitaria. Los factores de riesgo pueden ser familiares, sociales y personales y dentro de ellos podríamos incluir los problemas económicos y familiares, la cercanía a consumidores de alcohol u otras drogas, la insolidaridad social, los barrios marginales, la frustración por carencia de formación, la perdida de confianza en si mismo y el no poder superar las dificultades.

El consumo de alcohol en Europa es un problema de primera magnitud. La población en general no es consciente de los riesgos que entraña el consumo continuado o masivo de bebidas alcohólicas, y se le resta importancia debido a connotaciones sociales y culturales. La solución es compleja ya que los intereses políticos y comerciales interfieren en la aplicación de políticas eficaces que puedan llegar realmente a producir una reducción en el consumo y un decremento de los problemas relacionados con las bebidas alcohólicas.

La solución no está solo en manos de los estadistas, sino también en la concienciación de los distintos sectores sociales implicados, sanitarios, sociales y en la propia sociedad, que debe percibir inequívocamente las paradojas sobre las cuales se ha formado una cultura eminentemente pro - alcohólica.

Estos esfuerzos deben realizarse con más ímpetu en los países de la Europa del este y en los países en vías de desarrollo pues la pobre realidad social en la que se ven inmersos es ya por si misma un factor de riesgo asociado al incremento del consumo en los últimos años.

\section{BIBLIOGRAFÍA}

(1) Rodríguez Fernández, E. et al. Aproximación a los conocimientos de los médicos de atención primaria sobre el consumidor excesivo de alcohol y problemas relacionados. SEMERGEN. 2001; 27: 5-13.

(2) Conferencia ministerial de Estocolmo. OMS. Estocolmo- Suecia 19-02-2001.

(3) Produktschap Voor Gedistilleerde Dranken. World Drinks Trends: Henley - on - Thames, NTC Publications Ltd; 1996.

(4) Plan Nacional sobre Drogas. Memoria 1999. Ministerio del Interior. Imprenta Nacional B.O.E. Madrid. 2000.

(5) http://www.jornada.unam.mx/1999/jun99/ 990628/cien-zacarias.htm/

(6) Rubio Valladolid, G; Santo-Domingo Carrasco, J. Guía Práctica de Intervención en el alcoholismo. Madrid. 2000. P.429.

(7) Sánchez,A; Hidalgo, M.J. Efectos fetales del alcohol. El síndrome alcohólico fetal. En Tratado de Alcohología. Madrid. 2000. p.p. $285-$ 303. 
(8) Ministerio de Agricultura Pesca y Alimentación. La alimentación en España 1999. Sección General de Alimentación. http://www.mapya.es/

(9) Observatorio español sobre drogas. Informe $n^{\circ}$ 3.Plan nacional sobre Drogas. Imprenta Nacional B.O.E. Madrid. 2000.

(10) Navarro Botella, J. Aspectos sociológicos y epidemiológicos del consumo de alcohol en España en Tratado de Alcohología. Madrid. 2000. P. 443.

(11) F.A.R.E. XII Congreso Mundial de psiquiatría social. Washington D.C. Octubre 1990.

(12) El País: 20-4-2001, 22-4-2001, 15-5-2001, 12-62001.

(13) Portella y cols. El Alcohol y su abuso: Impacto socioeconómico. Edit. Panamericana. Madrid 1998.

(14) Ferrer, E. Un problema nacional: la dependencia alcohólica. Jano. 2-8 Julio 1999. Vol. LVII nº 1308.

(15) El Mundo: 21-3-1998, 2-11-1998, 11-11-1998, 16-11-1998, 16-12-1998, 16-2-1999, 24-1-1999, 9-5-1999, 27-6-1999, 9-7-1999, 20-7-1999, 2910-1999, 17-4-2000, 24-6-2000.

(16) Sánchez Mayka. MedSpain. Madrid 2001.

(17) http://estrelladigital.com/ (22-05-01)

(18) Bobes, J. et al. Estado actual de los alcoholismos. Psiquiatría biológica ( $n^{\circ}$ monográfico). Vol. 5, suplemento 1. Septiembre 1998.

(19) Gual Solé, A. et al. Alcoholismo. Jano. 20-26 octubre 2000. Vol. LIX. Nº 1361.

(20) Jano On - line, 31-10-2000.

(21) Observatorio Español sobre Drogas. Informe $n^{\circ} 2$. Ministerio del Interior. Delegación del Plan Nacional sobre Drogas. Imprenta Nacional B.O.E. Madrid. 2000.
(22) Rico Irles, J. El consumo de alcohol. Anales de medicina Interna. Vol.12. No 11 Madrid 1995.

(23) Crapelt, Michel. Young people's drinking and their environment. National Association for the prevention of Alcohol, París. France. Conference on Young People and Alcohol. O.M.S. World Health Organization. Regional Office for Europe. 2001.

(24) Rehn, N.; Room, R.; Edwards, G. Alcohol in the European Region - consumption, harm and policies. World Health Organization. Regional Office for Europe. 2001.

(25) Lay, William. Alcohol problems in the family: the scale of the problem and society's response. Confederation of family Organizations in the European Union (COFACE), Brussels, Belgium. Conference on Young People and Alcohol. O.M.S. World Health Organization. Regional Office for Europe. 2001.

(26) Zabelin, Pavel. Young people and alcohol in Russia. Director, Moscow Youth Centre. Russian Federation. Conference on Young People and Alcohol. O.M.S. World Health Organization. Regional Office for Europe. 2001.

(27) Heather, Nick. Brief interventions: An opportunity for reducing excessive drinking. Centre for alcohol \& Drug Studies. Newcastle City Health Trust \& University of Northumbria at Newcastle. United Kingdom. Conference on Young People and Alcohol. O.M.S. World Health Organization. Regional Office for Europe. 2001.

(28) Walburg, Jan A. Efficiency of treatment of dependence. Jellinek Clinics, Amsterdam Erasmus University. Rotterdam. Netherlands. Conference on Young People and Alcohol. O.M.S. World Health Organization. Regional Office for Europe. 2001. 